\title{
El magnesio no fue efectivo para el tratamiento de los calambres musculares nocturnos
}

\author{
Magnesium for the Treatment of Nocturnal Leg Cramps. A Crossover Randomized Trial \\ Frusso R, Zárate M, Augustovski F and Rubinstein A. J Fam Pract 1999 (Nov); 48: 868-871
}

\section{Objetivo}

Determinar la eficacia de un preparado farmacológicos con magnesio (citrato de magnesio) para el tratamiento de los calambres musculares nocturnos en miembros inferiores.

\section{Diseño}

Estudio prospectivo cruzado* randomizado doble ciego controlado con placebo.

\section{Lugar}

Servicio ambulatorio, Unidad de Medicina Familiar y Preventiva del Hospital Italiano de Buenos Aires, Argentina.

\section{Pacientes}

Se aleatorizaron 45 pacientes con historia de por lo menos 6 episodios de calambres nocturnos en el mes previo. La edad promedio fue de 61,6 años (rango de 28 a 87 ), el $73 \%$ de sexo femenino, el $24 \%$ fumadores, $43 \%$ hipertensos, $10 \%$ utilizadores de diuréticos, $19 \%$ portadores de várices en miembros inferiores, y 5\% diabéticos tipo 2 .

\section{Intervención \\ Luego de un periodo de lavado de medicación con placebo de cuatro semanas, todos los pacientes recibieron un mes de tratamiento activo y un mes de placebo. La secuencia de los periodos fue aleatoria. En el periodo de tratamiento activo se utilizó una dosis oral de $900 \mathrm{mg}$ de citrato de magnesio dos veces por día. Con este esquema, la mitad de los pacientes recibió primero el placebo y luego el magnesio; y la otra mitad recibió la secuencia inversa.}

\section{Medición de resultados principales}

Cuarenta y dos pacientes completaron los 4 meses de estudio. El resultado principal fue el número de calambres nocturnos en miembros inferiores. Los resultados secundarios fueron la duración, severidad, y trastornos del sueño causados por los calambres. Los episodios eran registrados por los pacientes en un diario autoadministrado.

\section{Resultados Principales}

Cuando los pacientes recibían placebo, el promedio mensual de calambres fue de $11,1 \pm 7,3$; mientras que al recibir magnesio el promedio fue de $11,8 \pm 7,6$ episodios. No hubo diferencias estadísticamente significativas entre ambos grupos $(p=0,59)$. Tampoco se registraron diferencias en la duración, severidad, y trastornos del sueño causados por los calambres. Se observó un significativo efecto periodo (periodeffect bias): todos los pacientes tuvieron una mejoría en el tiempo en cuanto al promedio de calambres nocturnos, independientemente de la intervención que recibieran. La severidad de los episodios, su duración y los trastornos del sueño, también mostraron una tendencia a la mejoría de período a período, si bien las diferencias no alcanzaron significación estadística.

\section{Conclusiones}

El magnesio no fue eficaz para el tratamiento de los calambres musculares nocturnos de miembros inferiores. Los pacientes mejoraron en el tiempo independientemente del tratamiento recibido.

\section{COMENTARIO}

Los calambres musculares o contracciones súbitas, bruscas y dolorosas de un músculo o grupo de músculos, suelen ocurrir en personas saludables, especialmente después de un ejercicio intenso. ${ }^{1}$ Sin embargo, algunos pacientes refieren calambres en miembros inferiores durante el sueño. Estos son en general inofensivos y no requieren tratamiento. No obstante, cuando se hacen frecuentes y alteran el descanso nocturno, pueden afectar la calidad de vida lo suficiente como para llevar al paciente a la consulta y ameritar una intervención médica. En nuestro medio es muy generalizado el uso de sales de magnesio para el tratamiento de los calambres nocturnos. Muchos pacientes refieren mejoría con esta terapéutica, pero la eficacia científicamente objetivable de este tratamiento no había sido documentada, a excepción de un ensayo en mujeres embarazadas. ${ }^{2}$ El otro fármaco conocidamente eficaz es el sulfato de quinina, ${ }^{3}$ si bien solo se recomienda en casos severos debido a su potencial toxicidad. También se han ensayado con diferente éxito, intervenciones no farmacológicas como los ejercicios de estiramiento y elongación muscular previos a irse a dormir.

El presente estudio proporciona enriquecedora información. Se trata de un estudio prospectivo aleatorizado a doble ciego y controlado, diseño que aporta la mejor evidencia para ensayos intervencionales. El estudio contaba con un buen poder* para detectar dife- rencias clínicamente relevantes, por lo que parecen confiables sus conclusiones. La medición del resultado principal (número de calambres) se hacía a través de un diario que llenaba el paciente diariamente. A pesar que pudo haber algún olvido, estos deberían distribuirse aleatoriamente y no interferir en los resultados observados.

El diseño cruzado de la intervención minimizaría un potencial disbalance azaroso de pacientes más susceptibles en alguno de los grupos, ya que todos los pacientes recibieron las 2 intervenciones (droga y placebo). Los hallazgos revelaron que el magnesio no es eficaz en el tratamiento de los calambres nocturnos comparado con placebo. Resalto esto último por la importante referencia al efecto periodo observado en el estudio. Como ocurre con otros trastornos a los que podríamos llamar "funcionales", sin sustrato orgánico, se advirtió una paulatina mejoría en todos los pacientes, independientemente de la intervención recibida. Si bien como señalan los autores, esto puede deberse a la variabilidad natural de la condición, fenómeno de regresión a la media o genuino efecto placebo, la conclusión final del estudio podría reformularse en los siguientes términos: es aconsejable la utilización de placebo en el tratamiento de los calambres nocturnos; las sales de magnesio no serían más efectivas que cualquier otro placebo.

*Ver Glosario

\section{Dr. Alejandro Regueiro}

Director Médico de S.P.M.

Referencias

1. McGee SR. Muscle cramps. Arch Intern Med 1990; 150: 511-518.

2. Dahle LO, Berg G, Hammar M, et al. The effect of oral magnesium substitution on pregnancy-induce leg cramps. Am J 0bstet Gynecol 1995; 173: 175-180.

3. Connolly PS, Shirley EA, Wasson JH, et al. Treatment of nocturnal leg cramps: a crossover trial of quinine vs. vitamin E. Arch Intern Med 1992; 152: $1877-1880$. 